\title{
Serotonin syndrome caused by overdose with paroxetine and moclobemide
}

\author{
C R FitzSimmons, S Metha
}

\begin{abstract}
Well known clinical syndromes can be produced by overdose with more commonly ingested substances such as opiates or tricyclic antidepressants. A case of a much more unusual syndrome presenting to the accident and emergency department resulting from overdose with a combination of tablets is reported. The clinical presentation of serotonin syndrome and its management are described. This resulted from acute ingestion of paroxetine, a selective serotonin reuptake inhibitor, and moclobemide, a monoamine oxidase inhibitor.

(F Accid Emerg Med 1999;16:293-295)
\end{abstract}

Keywords: serotonin syndrome; paroxetine; moclobemide

\section{Case report}

A 28 year old man attended the accident and emergency (A\&E) department three hours after taking an overdose of 12 paracetamol $(6 \mathrm{~g}), 30$ paroxetine tablets $(600 \mathrm{mg})$, and an unknown quantity of a third unidentified tablet. He denied taking any alcohol or any other drugs.

On arrival he was alert, orientated, and talkative. History revealed no medical problems other than chronic lower back pain. $\mathrm{He}$ reported that he had vomited twice, one hour after the ingestion of the tablets. No tablet particles were seen.

On examination he was anxious and had a mild tremor but was fully orientated. His pulse was 80 beats $/ \mathrm{min}$ and regular, blood pressure $140 / 80 \mathrm{~mm} \mathrm{Hg}$, and heart sounds were normal. He had a respiratory rate of 30 breaths/min and the chest was clinically clear. There were no other physical findings of note. After discussion with the regional poisons' centre it was advised that doses of paroxetine less than $600 \mathrm{mg}$ were relatively safe and this needed no treatment other than activated charcoal and observation for at least six hours. Blood was taken for paracetamol and salicylate concentrations at this point.

Shortly after this the patient became very excitable. He began to sweat and tensed up all the muscles of both legs. Agitation gave way to an increasingly confused state. Nystagmus developed and this led on to a full oculogyric crisis. Procyclidine $10 \mathrm{mg}$ was given intramuscularly and repeated after 20 minutes but with no effect.

Over this period his condition deteriorated alarmingly. His Glasgow coma score fell from 15 to 7 and he became increasingly tremulous until almost all muscle groups were involved.
There was generalised involuntary twitching of all limbs and especially the face and jaw. On opening the eyes both pupils were dilated to 7 $\mathrm{mm}$ diameter but were equal and reacting to light. All reflexes were extremely brisk and the plantar reflexes were extensor.

The blood pressure increased to $160 / 100$ $\mathrm{mm} \mathrm{Hg}$ and the heart rate increased to 200 beats/min and remained regular. Profuse sweating continued and the axillary temperature was recorded at $37.6^{\circ} \mathrm{C}$.

At this point the patient was transferred to the resuscitation room; $100 \%$ oxygen was administered and cardiac monitoring begun. Chest radiography and electrocardiography (ECG) were performed, a test strip was used to measure blood glucose, and blood samples were sent for culture, full blood count, urea and electrolytes, liver function tests, calcium, magnesium, creatinine kinase, glucose, and toxicology. There was no clinical evidence to suggest disseminated intravascular coagulation and clotting studies were not performed in the A\&E department. The patient was catheterised and urine sent for toxicology and myoglobin concentrations. Repeat axillary temperature read $39.8^{\circ} \mathrm{C}$ and the patient continued to sweat profusely. Core temperature was not recorded in the $A \& E$ department.

Anaesthetic assistance was sought to paralyse and ventilate the patient as muscular rigidity began to cause respiratory embarrassment. Shortly before muscular relaxation was induced the patient developed opisthotonus and became rigid in the facial muscles with trismus. His blood pressure fell to $80 / 50 \mathrm{~mm}$ $\mathrm{Hg}$ and he came close to respiratory arrest with very shallow respirations at a rate falling to 4 breaths/min.

At this point a differential diagnosis was made of neuroleptic malignant syndrome; ingestion of ecstasy; sepsis; meningitis; encephalitis; or intracranial haemorrhage based on the clinical findings alone. There was no obvious clinical reason to suspect tetanus.

Intravenous cefotaxime ( $2 \mathrm{~g})$ was given, an intravenous infusion of colloid started, and arrangements made for urgent computed tomography of the head. Blood pressure normalised within five minutes of muscular paralysis, although the pulse rate remained 200 beats/ min and regular.

Discussion with the patient's parents revealed no further relevant history and they were requested to return to the patient's flat to search for any evidence of other tablets not found by the ambulance personnel.

Computed tomography gave a normal result and the patient was taken to the intensive care 
unit (ICU). In the meantime the parents returned to the hospital with an empty bottle of moclobemide that had been overlooked. A definitive diagnosis of serotonin syndrome was then made.

Results of all blood tests including clotting studies performed in the ICU were normal. Blood glucose by test strip was $7.0 \mathrm{mmol} / 1$. Chest radiography was normal and ECG confirmed a sinus tachycardia. Results of urine and blood cultures were negative. Core temperature was measured at $40.2^{\circ} \mathrm{C}$ and fell to $37.0^{\circ} \mathrm{C}$ within hours of paralysis and sedation. Toxicology later revealed the presence of paroxetine, moclobemide, and the procyclidine given in the $\mathrm{A} \& \mathrm{E}$ department.

After 24 hours of supportive ICU treatment the patient made an uneventful recovery. $\mathrm{He}$ remained an inpatient for three days due to expression of suicidal thoughts and a comprehensive psychiatric assessment was made. Since discharge he has remained well.

\section{Discussion \\ SEROTONIN}

Serotonin (5-hydroxytryptamine/5-HT) is generated by the metabolism of dietary tryptophan. It is stored in intracellular vesicles until neuronal depolarisation causes release of the neurotransmitter into synapses. Monoamine oxidase (MAO) metabolises any serotonin not protected within vesicles so MAO inhibitors allow concentrations to rise. Once released into a synapse the action of serotonin is rapidly terminated by its presynaptic reuptake. The block to this reuptake is the site of action of the selective serotonin reuptake inhibitors (SSRIs) and the combination of a MAO inhibitor and a SSRI will obviously lead to very raised concentrations of serotonin in the synaptic cleft via the two separate mechanisms.

\section{OVERDOSE WITH SELECTIVE SEROTONIN}

REUPTAKE INHIBITORS

Overdose with SSRIs such as fluoxetine (Prozac), paroxetine (Seroxat), and fluvoxamine are considered to be relatively safe in comparison with other antidepressants such as tricyclic antidepressants. ${ }^{1}$ Fifty per cent of patients are asymptomatic, though others may present with tachycardia, nausea, vomiting, tremor, and drowsiness. Such symptoms are generally benign and of short duration. Doses of up to $3.6 \mathrm{mg} / \mathrm{kg}$ would appear to cause little or no symptoms and even higher doses are relatively safe unless potentiated by alcohol. ${ }^{2-4}$ The SSRIs are selective inhibitors of the presynaptic 5-HT reuptake transporter. ${ }^{5}$ As this is a highly selective process SSRIs have considerably fewer side effects both at therapeutic and toxic concentrations compared with tricyclic antidepressants which also bind nonspecifically to muscarinic, histaminergic, dopaminergic, and adrenergic receptors. ${ }^{6-8}$

Experience with these relatively new drugs in overdose is limited, one reason being that they are commonly taken with other drugs, which makes interpretation of the effects of the SSRI alone difficult. ${ }^{1}$
SEROTONIN SYNDROME

The serotonin syndrome results from serotonergic hyperstimulation in the brainstem and spinal cord and may very closely mimic neuroleptic malignant syndrome. ${ }^{6}$ It is generally short lived but early recognition and adequate supportive treatment are necessary. The syndrome is characterised by alteration in mental state (confusion, agitation, coma); abnormal motor activity (myoclonus, rigidity, hyper-reflexia, incoordination); autonomic instability (fever, sweating, diarrhoea, tachycardia, labile blood pressure, pupillary dilatation); and can also cause nystagmus, seizures, oculogyric crises, opisthotonus, myoglobinuria, renal failure, disseminated intravascular coagulation, cardiac arrhythmias, adult respiratory distress syndrome, coma, and death. ${ }^{6}$

Serotonin syndrome occurs when the SSRIs are taken in combination with other antidepressants such as tricyclic antidepressants and MAO inhibitors but can also occur by altering one type of SSRI to another. The SSRIs have a prolonged washout phase and interacting drugs should not be started for at least five weeks after coming off SSRIs. Likewise SSRIs should not be started until two weeks after stopping MAO inhibitors. Pethidine, pentazocine, carbamazepine, and dextromethorphan also block presynaptic reuptake of serotonin and these serotomimetic agents can interact with SSRIs to cause serotonin syndrome. ${ }^{6}$

The onset of the syndrome can range from minutes after initiating the second agent to weeks after being on a stable dosage. ${ }^{8}$ The combination of a MAO inhibitor and SSRI is the most commonly implicated in the pathogenesis of the serotonin syndrome, the SSRI most often being fluoxetine due mainly to its popularity and wide prescribing. ${ }^{8} \mathrm{Mo}-$ clobemide seems to be particularly involved in fatal outcome, having been the MAO inhibitor implicated in at least six such cases. ${ }^{9} 10$ There have been only two documented cases of serotonin syndrome caused by a single agent. ${ }^{8}$

\section{DIAGNOSIS}

The following three criteria suggested by Sternbach must be fulfilled to diagnose serotonin syndrome ${ }^{11}$ :

(1) At least three of the following signs and symptoms: agitation, hyper-reflexia, sweating, shivering, tremor, diarrhoea, hyperthermia, or mental state changes (confusion, hypomania).

(2) Symptoms must be associated with an initiation or an increase in the dose of a serotomimetic agent.

(3) There must also be exclusion of other causes such as infection, metabolic disturbance, substance abuse/withdrawal, or the use of neuroleptic agents.

\section{MANAGEMENT}

The management of overdose with SSRIs, as previously stated, involves little more than supportive treatment. A full history should be taken including the number, type, and strength of all tablets taken and the timing of ingestion. A past medical history, history of any allergies, and a current drug history can prove very use- 
ful. Specific inquiry should be made about drugs known to interact with SSRIs, including alcohol. Examination of patients is unlikely to yield any abnormality but an assessment of mental state is essential and early subtle signs of a significant overdose may be elicited in the neurological examination such as tremor, nystagmus, and dyskinesias. A full assessment of suicidal risk should always be performed.

Investigation should initially include baseline full blood count, urea and electrolytes, glucose and concentrations of paracetamol and salicylates since these are commonly taken with other drugs in overdose and good guidelines exist for treatment should concentrations be raised. There is no routine test for measuring concentrations of SSRIs currently available. ECG is advisable as arrhythmias can be precipitated and monitoring should be started if any abnormalities are found.

Treatment is supportive and includes stopping/removing the suspected drug. Activated charcoal can be given and reduces the absorption of tablets from the stomach. SSRIs are not known to delay gastric emptying so its use cannot be advocated with presentation after two hours from ingestion. Antiemetics can be given for nausea and vomiting. Gastric lavage is indicated if a significant dose has been taken and presentation is within two hours, leaving activated charcoal in the stomach afterwards.

Hyperthermia can be treated by active cooling or by the use of chlorpromazine which has both antipyretic and sedative properties. Intravenous fluid replacement is essential to correct losses from excessive sweating, prevent cardiovascular collapse, and augment active cooling. Dantrolene may be useful as in the neuroleptic malignant syndrome or malignant hyperpyrexia syndrome with which serotonin syndrome has many related features. Clonazepam or diazepam can be used both for severe myoclonus or for control of seizures. Nifedipine is useful for hypertensive episodes and in severe cases sedation, paralysis, and ventilation may be required. The use of serotonin antagonists such as methysergide and cyproheptadine would appear logical and has met with some clinical success. ${ }^{8}$ Both are non-specific 5-HT1 and 5-HT2 receptor antagonists. Bromocriptine, chlorpromazine, and dantrolene have been used with inconsistent results. ${ }^{6}$

Mortality may be caused by rhabdomyolysis, disseminated intravascular coagulation, adult respiratory distress syndrome, and cardiovascular collapse, all secondary to the hypermetabolic state resulting from myoclonus or muscular rigidity.

\section{CONCLUSION}

Newer antidepressants such as the SSRIs are generally regarded as safe in overdosage when compared with the more commonly taken tricyclic antidepressants. This would appear to be the case when they are taken alone. However, in combination with certain other drugs, notably other antidepressants like MAO inhibitors, there are very significant side effects and the potential to develop the serotonin syndrome. This has been documented to be fatal in individual cases. We believe this to be only the second case of the serotonin syndrome to present after ingestion of paroxetine and moclobemide and the first to have a successful outcome. The case highlights the clinical features of a potentially fatal combination. Serotonin syndrome should be considered in any patient presenting to an $A \& E$ department with the features described.

Conflict of interest: none.

Funding: none.

1 Singer PP, Jones GR. An uncommon fatality due to moclobemide and paroxetine. $\mathcal{f}$ Anal Toxicol 1997;21:51820 .

2 Kincaid RR, McMullin MM, Crookham SB, et al. Report of a fluoxetine fatality. $\mathcal{F}$ Anal Toxicol 1990;14:327-9.

3 Clement GE, Sell J, Mihalakis I, et al. Fluoxetine fatal poisoning - a case report. $\mathcal{F}$ Anal Toxicol 1996;20:68-9.

4 Rohrig TP, Prouty RW. Fluoxetine overdose: a case report. $\mathcal{F}$ Anal Toxicol 1989;13:305-7.

5 Gram LF. Fluoxetine. N Engl f Med 1994;331:1354-61.

6 Kam PCA, Chang GWM. Selective serotonin reuptake inhibitors. Anaesthesia 1997;52:982-8.

7 Finley PR. Selective serotonin reuptake inhibitors: pharmacological profiles and potential distinctions. Ann Pharmacother 1994;28:1359-69.

8 Sporer KA. The serotonin syndrome. Drug Saf 1995;13:95104.

9 Neuvonen PJ, Pohjola-Sintonen S, Tacke U, et al. Five fatal cases of serotonin syndrome after moclobemide-citalopram or moclobemide-clomipramine overdoses. Lancet 1993; 342:1419-23.

10 Spigset O, Mjorndal T, Lovheim D. Serotonin syndrome caused by a moclobemide-clomipramine interaction. $B M \mathcal{F}$ 1993;306:248-50.

11 Sternbach $\mathrm{H}$. The serotonin syndrome. Am $\mathcal{f}$ Psychiatry 1991;148:705-13. 